\title{
EDUCACIÓN MEDIA SUPERIOR: UNA VÍA DE EMPODERAMIENTO PARA LA MUJER INDÍGENA
}

\section{HIGH SCHOOL: A PATH OF EMPOWERMENT FOR INDIGENOUS WOMEN}

\author{
Mtra. Verónica Tamayo Arguello ${ }^{1}$ \\ Dra. Ivett Reyes Guillén ${ }^{2}$ \\ Dra. Socorro Fonseca Córdoba ${ }^{3}$ \\ México
}

\begin{abstract}
1 ve_k_e@hotmail.com
(992) $110464 \overline{3}$ Instituto de Estudios Superiores Emilio Rabasa Estebanell San Cristóbal de Las Casas, Chiapas, México Maestra en Educación por el Instituto de Estudios Superiores Emilio Rabasa Estebanell. Ha publicado capítulo de libro de la Red de Investigación en Salud Pública y Atención a problemas del Desarrollo. Mtra. Verónica Tamayo Argüello. ORCID https://orcid. org/0000-0002-1189-8907

$2 \quad$ ivettrg017@gmail.com (967) 671953137

Facultad de Ciencias Sociales, Universidad Autónoma de Chiapas, San Cristóbal de Las Casas, Chiapas, México. Google Académico: https://scholar.google.es/scholar?hl=es\&as sdt=0\%2C5\&q=ivett+reyes-guill\%C3\%A9n\&oq=ivett+reye

ORCID https://orcid.org/0000-0001-9738-4554

Doctora y Maestra en Ciencias por EI Colegio de la Frontera Sur, ECOSUR. Profesor de tiempo completo de la Universidad Autónoma de Chiapas. Perfil deseable PRODEP. Integrante del CA-UNACH-157 PRODEP. Presidente de la Red de Investigación en Salud Pública y Atención a problemas del Desarrollo. Ha publicado en 13 libros en temáticas tales como violencia en el proceso enseñanza-aprendizaje, clima organizacional., género. Los más recientes artículos indexados internacionales publicados en International Journal of Innovative Science and Research Technology; International Journal of Humanities Social Sciences and Education (IJHSSE).

3 socorrofonseca102@hotmail.com 967)6781630 Facultad de Ciencias Sociales, Universidad Autónoma de Chiapas ve_k_e@hotmail.com (992)1104643 Instituto de Estudios Superiores Emilio Rabasa Estebanell, San Cristóbal de Las Casas, Chiapas, MéxicoDoctora en Educación por el Instituto de Estudios Universitarios. Profesor de tiempo completo de la Universidad Autónoma de Chiapas. Perfil deseable PRODEP. Líder del CA-UNACH-157 PRODEP. Integrante de la Red de Investigación en Salud Pública y Atención a problemas del Desarrollo. Ha publicado y coordinado libros dentro del a Red de Investigación en Salud pública y atención a problemas del Desarrollo. Los más recientes artículos indexados internacionales publicados en International Journal of Innovative Science and Research Technology; International Journal of Humanities Social Sciences and Education (IJHSSE). Dra. Socorro Fonseca Córdoba. Google Académico https://scholar.google.es/scholar?start=10\&q=SOCORRO+FONS ECA+CORDOBA\&hl=es\&as_sdt=0,5 ORCID https://orcid.org/0000-0003-1885-1914
\end{abstract}




\section{RESUMEN}

El tema de educación nos abre un abanico de posibilidades para analizar; hasta nuestros días no deja de sorprendernos con adecuaciones y nuevas reformas. En lo que respecta a la educación indígena el panorama se torna complejo, dado que la institucionalización de la educación se diseñó para una población monolingüe no indígena, siguiendo los patrones de un sistema que nos obliga a ver a la educación como un escalón para alcanzar el desarrollo humano y social. En la presente investigación se indagó y analizó la manera en que la población de estudio, mujeres indígenas nivel media superior, se perciben y construyen dentro del proceso de educación.

PALABRAS CLAVE: Mujeres indígenas, empoderamiento, educación

\section{ABSTRACT}

The topic of education opens a range of possibilities for discussion; to this day, it never ceases to amaze us with adaptations and new reforms. About indigenous education, the picture becomes complex, as the institutionalization of education was designed for a non-indigenous monolingual population, following the patterns of a system that forces us to see education as a step to achieve human and social development. This research investigated and analyzed how the study population, upper middle indigenous women, are perceived and built within the education process.

KEY WORDS: Indigenous Women,

Empowerment, Education

\section{INTRODUCCIÓN}

Para la presente investigación, tomaremos como punto de partida una breve explicación del nivel educativo sobre el cual se trabajó. El nivel medio superior a cargo del Colegio de
Bachilleres de Chiapas, México, actualmente maneja dos modalidades: 1) planteles $\mathrm{COBACH}$ ubicados en su mayoría en ciudades y las cabeceras municipales en donde la demanda educativa es alta; 2) planteles EMSaD, Educación Media Superior a Distancia) esta modalidad tiene presencia en ejidos y comunidades indígenas en donde la demanda educativa es menor. Actualmente el Colegio de Bachilleres de Chiapas, México, cuenta con un total de 330 centros educativos de los cuales la mayor parte son centros EMSaD, distribuidos por toda la entidad, Chiapas es una zona de alta marginación socioeconómica y con graves problemas de desarrollo.

El estudio se realizó en un EMSaD 315 ubicado en la comunidad de Iwiltic, dentro del Municipio de San Juan Cancúc, Chiapas, México, durante el año 2019. La población total de Iwiltic es de 784 personas, de cuales 383 son masculinos y 401 femeninas. Dentro de los habitantes de Iwiltic, 784 personas viven en hogares indígenas. Son bilingües, de idioma indígena tzotzil y español, 346 personas. Monolíngües de habla tzotzil 293, los de cuales hablan también mexicano es 346. Esta localidad se encuentra en un municipio y una zona de Muy Alta marginación socioeconómica, Región VI Selva (CONAPO, 2010).

\section{EL CONCEPTO DE INDIGENISMO $Y$ SU APORTACIÓN A LA EDUCACIÓN INDÍGENA.}

El término indigenismo surge en 1921 como resultado de un planteamiento político en el que se pretendía reivindicar la participación de la población indígena al plano nacional. Por su parte José Vasconcelos, siendo el primer titular de la Secretaria de Educación, es quien integra la política indigenista al ámbito educativo, de acuerdo con Dietz (2014), la política indigenista tuvo como principio básico incluir a la población indígena al ámbito social, ya que históricamente 
han sido excluidos en muchos aspectos de la vida social de nuestro país. Sobre el termino indigenismo, Dietz lo describe desde la postura política como: el proyecto de mestizaje nacional implica medidas específicamente destinadas a "integrar" a aquella población en el Estadonación mestizo que aún no son y/o no se consideran mestizos: los indígenas."

En cuanto a materia educativa, la población indígena pudo tener acceso a la educación; pero no de la manera que se hubiera querido ya que por su condición de indígenas la mayor parte de las veces han sido excluidos o no han tenido las mismas oportunidades que la población no indígena.

Aunque en sus intentos por incluir a la población indígena a la política nacional se han hecho algunas adecuaciones en materia educativa como lo es el hecho de la educación intercultural o bilingüe, aún falta trabajar mucho para lograr cambios significativos en la vida de la población indígena.

El enfoque de género como perspectiva de análisis. La categoría de género tiene sus inicios con Simone de Beauvoir en 1949, al afirmar que "una no nace si no que se hace mujer", convirtiéndose en la primera estudiosa en analizar la inequidad entre los sexos, por lo tanto, plantea que las características humanas consideradas como femeninas son adquiridas por las mujeres mediante un complejo proceso individual y social en lugar de derivarse naturalmente de su sexo (Lamas,1996). La categoría de género se convirtió rápidamente en uno de los cimientos conceptuales con que las feministas construyeron sus argumentos políticos.

Lamas (2003), nos menciona que el concepto de género ha abierto un espacio de análisis dentro del campo de las ciencias sociales. Cada vez se reconoce más al género como una categoría útil y necesaria para la lectura, interpretación y explicación de los comportamientos humanos, abriendo posibilidades dentro de la investigación social.

No podemos afirmar que el concepto pertenece únicamente a la perspectiva feminista, actualmente el concepto de género ha permitido desarrollar una amplia perspectiva teórica con vías de inclusión y desarrollo. Con el concepto de género se ha abierto un campo de análisis, el cual nos orienta a hacer visibles las desigualdades y las condiciones de vida de las mujeres con respecto a los hombres; estas desigualdades se hacen presentes en diversos ámbitos, tales como lo político, lo social y lo económico.

El concepto de género no es aplicable únicamente al ámbito femenino, este concepto también puede dar pauta al análisis del comportamiento masculino, como bien lo señala Scott (2003), el mundo de las mujeres es parte del mundo de los hombres creado en él y por él. Con este enfoque se trata de explicar las diferencias construidas socialmente a partir de la diferencia sexual; es decir que la misma sociedad nos adjudica roles que debemos desempeñar, estos roles van de acuerdo con nuestro sexo y edad. Es claro que esta perspectiva nos permite analizar las relaciones entre hombres y mujeres aceptando que estas parten de las desigualdades en todos los aspectos para las mujeres, siendo los hombres quienes han dominado a las mujeres históricamente.

Por su parte García (2000), define el concepto de género como la construcción sociocultural de la diferencia sexual, aludiendo con ello al conjunto de símbolos, representaciones, reglas, normas, valores y prácticas que cada sociedad y cultura elabora colectivamente a partir de las diferencias culturales de hombres y mujeres.

Para culminar el apartado de género y citando a Solís tenemos que... 
"Es muy importante definir la palabra género y diferenciarla de la de sexo. El sexo es biológico y contiene cualidades físicas $y$ anatómicas que se traducen en la distinción biológica entre hombres y mujeres. Al contrario, el género, o en otras palabras el sexo social, es una construcción, es decir, no tiene casi nada que ver con características físicas y ni con la genética. Utilizando ésta como base diríamos que las diferencias biológicas construyen diferencias sociales que afectan a la vida social y que crean ciertos papeles y expectativas de comportamiento" (Solís, 2016:100).

Género y educación. La perspectiva de género nos permite comprender de qué manera se construyen estas diferencias entre hombres y mujeres. Podemos también analizar las formas en que las jóvenes indígenas viven y experimentan diariamente está marcada diferencia y de qué manera repercute en diferentes aspectos de su vida; en particular dentro del ámbito educativo.

Hablemos primeramente de educación y cómo esta es transmitida por medio de la escuela que actúa como un agente socializador, en la cual se debe transmitir conocimientos, reafirmar valores, establecer normas de comportamiento, desarrollar habilidades y destrezas que les servirán para la vida en sociedad de los estudiantes.

Históricamente la mujer ha sido excluida de diversos aspectos de la vida social, anteriormente las mujeres no tenían acceso a la educación de la misma manera que los hombres. De acuerdo con Montero (2000), la educación para las mujeres durante la época del México independiente estaba a cargo de una escuela de artes y oficios donde instruían y preparaban a la mujer para la vida en matrimonio. Eran muy pocos los colegios donde enseñaban a las mujeres a leer y escribir ya que esto era visto como algo malo porque despertaba "la malicia femenina". De acuerdo con la autora los datos respecto al papel de la mujer en el ámbito educativo se registran hasta el año de 1907 cuando Manuela Mota, se convierte en la primera mujer estudiante de preparatoria de la cual se tiene registro.

Datos más actuales del Instituto Nacional de Estadística y Geografía (INEGI, 2010), mencionan que actualmente existe un porcentaje significativo de mujeres y niñas que se encuentran cursando algún nivel educativo.

El papel de la escuela es asumir el rol de agente de cambio en la sociedad, considera que tanto escuela como familia son importantes agentes de socialización, ambos tienen el encargo social de educar en la cultura de la paz y la igualdad de género, construyendo conjuntamente valores y patrones no sexistas en las personas. Los actores de la educación debemos por medio del lenguaje, el trato, los juegos, enseñanzas y otras prácticas que refuercen los estereotipos equivocados de género (Solís, 2016).

Por lo anterior, y por el sentido que damos a este artículo, consideramos la definición de educación de (Araya, 2001: 197):

“El concepto de educación es más amplio que el de enseñanza y aprendizaje, y tiene fundamentalmente un sentido espiritual y moral, siendo su objeto la formación integral del individuo. Cuando esta preparación se traduce en una alta capacitación en el plano intelectual, en el moral y en el espiritual, se trata de una educación auténtica, que alcanzará mayor perfección en la medida que el sujeto domine, autocontrole $y$ 
autodirija sus potencialidades:

deseos, tendencias, juicios, raciocinios y voluntad. La educación es el conjunto de conocimientos, órdenes y métodos por medio de los cuales se ayuda al individuo en el desarrollo y mejora de las facultades intelectuales, morales y físicas. La educación no crea facultades en el educando, sino que coopera en su desenvolvimiento y precisión".

\section{EDUCACIÓN Y EMPODERAMIENTO DE LAS MUJERES}

De acuerdo con Sánchez (2018), define la educación en este sentido como:

"La educación desempeña un papel decisivo en la emancipación de la mujer, la protección de los niños contra la explotación laboral, el trabajo peligroso y la explotación sexual, la promoción de los derechos humanos y la democracia, la protección del medio ambiente y el control del crecimiento demográfico"

(Sánchez, 2018:4).

La educación es el medio por el cual se transmiten conocimientos y valores, pero ¿Qué papel juega la educación en la construcción de género? El género y los estereotipos que emanan de él, también pueden ser transmitidos por medio de diversos procesos educativos.

"Los estereotipos de género y las relaciones entre los sexos, muchas veces jerárquicas, se reproducen en los procesos educativos, tanto dentro como fuera de los centros de enseñanza. En estos ámbitos se desarrollan aún más y se afianzan las identidades en relación con el género, por lo que la educación puede aportar al cuestionamiento de los modelos de pensamiento y a los roles estereotipados y discriminadores $y$, por ende, a romper con los patrones tradicionales y a superar los prejuicios" (Sánchez, 2018:5).

Si bien es cierto que, dentro de este proceso educativo, formal o informal se pueden llegar a concretar estereotipos de género equivocados, no debemos olvidar la verdadera función de la educación, formar personas críticas, analíticas y libres y claro que el género femenino no debe ser la excepción.

Por último, en este documento, consideramos al empoderamiento como lo describe (Sánchez, 2018:7): "Empoderarse significa que las personas adquieran el control de sus vidas, logren la habilidad de hacer cosas y de definir sus propias agendas. $Y$ por esto, resulta de vital importancia privilegiar el empoderamiento de las mujeres en el campo de la educación. Ese empoderamiento entendido como la acción que implica que las personas, y en este caso las mujeres, se conviertan en agentes activos como resultado de un accionar que varía de acuerdo con cada situación concreta. En este sentido, el empoderamiento es un proceso para cambiar la distribución del poder, tanto en las relaciones interpersonales como en las instituciones de la sociedad".

\section{METODOLOGÍA}

El presente estudio se realizó con un grupo de 26 mujeres, jóvenes estudiantes del EMSaD 315 Iwiltic, San Juan Cancúc, Chiapas, México, durante el año 2019. Se ubica dentro de la metodología y las técnicas cualitativas, observación y entrevistas a profundidad.

Por medio de la técnica de observación, se identificaron situaciones y acciones cotidianas 
de las estudiantes que permiten comprender de qué manera ellas perciben su estancia en la escuela. Se identificaron también elementos de carga cultural que en muchas ocasiones las limita en su desenvolvimiento dentro del ámbito educativo.

Así también, se utilizó a la entrevista a profundidad como medio de recolección de información. Estas entrevistas permitieron conocer de qué manera se construyen estas percepciones, así como los elementos tanto culturales y sociales que determinan la construcción de la imagen de la joven estudiante de nivel medio superior y el empoderamiento consecuente.

\section{DE LO ENCONTRADO EN EL ESTUDIO}

Se realizaron un total de 26 entrevistas a mujeres estudiantes de nivel medio superior en EMSAD, con una edad promedio de 17 años.

Lo que representa la educación para ellas. El $31 \%$ de las entrevistadas mencionaron que ven a la educación como una oportunidad para aprender. Según las jóvenes entrevistadas el hecho de ir a la escuela les permite aprender a leer, escribir, tener nuevos conocimientos, valores y nuevas ideas. Dicho en palabras de la informante No.16 : "Yo pienso que la educación es muy importante para mí porque me permite conocer y aprender muchas cosas y adquirir nuevos valores y conocimientos, la educación me sirve para prepararme y seguir adelante"

Otro $31 \%$ de las entrevistadas, mencionó que ven a la educación como algo importante, porque les ayuda a desarrollarse; pero sobre todo ven en la educación como oportunidad para salir adelante en su vida. Se refiere a que muchas de ellas quisieran salir de su comunidad y encontrar un trabajo que las haga independientes, así lo expresa textualmente la informante No. 10: "Yo pienso que la educación es buena porque me ayuda a mejorar mi futuro"

Las jóvenes piensan que la educación les permite prepararlas todos los días. El $26 \%$ de las estudiantes consideran que la educación es buena y manifiestan el hecho de que les gusta ir a la escuela porque es un lugar donde pueden aprender; es también el único lugar donde ellas pueden jugar y pueden ser tratadas de manera igualitaria.

El $12 \%$ de las entrevistadas, manifiesta que es importante porque aparte de adquirir conocimientos y desarrollar habilidades como la lectura y la escritura, les permite conocer sobre el mundo y sus derechos:

"Para mí, la educación en las mujeres es algo bueno, porque con el estudio nos preparamos para conocer más de nuestro mundo y tener más conocimientos" (Entrevistada No. 24)

"Yo pienso que el estudio me sirve para salir adelante, para aprender cosas nuevas, para saber que son los derechos que tengo, porque yo también tengo derechos, aunque soy mujer" (Entrevistada No. 25)

"Como mujer yo digo que es bueno prepararnos, porque también nosotras debemos de salir adelante, porque sabemos que también nosotras tenemos derechos tanto como los hombres. No debemos permitir cuando los hombres nos aplastan, debemos de defendernos, porque nosotras como mujeres también tenemos derechos" (Entrevistada No. 26)

En cuanto a la importancia que les representa la educación media superior (EMSaD): El 96\% de ellas manifestó que 
consideran a la educación media superior como algo muy importante; algunas ven en este nivel educativo una oportunidad de aprender cosas nuevas, otras mencionan que la educación media superior les da la oportunidad de continuar con sus estudios universitarios. Así también, piensan que les ayudará a conseguir un empleo, o bien, que les ayudará a cambiar patrones repetitivos de la cultura machista de la comunidad. Informante No. 5: "Si es importante porque se aprenden muchas cosas para salir adelante después"

La mayoría de estas chicas manifiesta que la educación media les permite desarrollar un plan de vida diferente al que están acostumbradas la mayor parte de las mujeres en la comunidad. Estas jóvenes tienen una visión diferente. Incluso algunas de ellas mencionan que la educación media superior les ayudará a continuar con una carrera universitaria o bien, a conseguir un trabajo en la ciudad, informante No. 8: "Si es muy importante para mi el $\mathrm{COBACH}$, porque si no estudiamos no vamos a poder saber que vamos a hacer en nuestro futuro y si estudiamos el COBACH vamos a prepararnos, educarnos y vamos a tener una idea de qué debemos hacer y también conocer nuestros derechos"

Informante No. 9: "Si, es importante porque nos ayuda a salir adelante en la vida, porque si no estudiamos no podemos salir a donde queremos y tampoco cambiar la costumbre de nuestra comunidad de cómo vivir la vida". Informante No. 24: "Para mí es importante porque me da herramientas en mi futuro y poder saber cuáles son las cosas que puedo hacer y también porque ahi me dicen sobre mis derechos"

\section{INFLUENCIA DE FACTORES CULTURALES.}

Ahora bien, respecto a la forma en que la cultura influye de manera positiva o negativa en la educación de estas jóvenes, iniciaremos contextualizando a las jóvenes entrevistadas; pertenecen a la etnia indígena tseltal, esta comunidad indígena se caracteriza por vivir una vida regida por usos y costumbres, los cuales indudablemente influyen en la vida de las mujeres de la comunidad.

El $81 \%$ de las entrevistadas manifestó que su cultura influye de manera negativa en su educación ya que se les obliga a vivir conforme a lo que rigen los usos y costumbres; en específico las limita de manera significativa en su educación, no tienen las mismas oportunidades para estudiar que los hombres, esto se puede corroborar con datos que se manejan en el control escolar del EMSaD, actualmente se cuenta con una matrícula total de 158 alumnos, de los cuales 103 son hombres y solo 55 son mujeres.

El argumento más escuchado es que las mujeres no deben estudiar porque ellas deberían mejor prepararse para ser amas de casa y para ello deben aprender a cocinar, lavar, atender al esposo. Dentro de esta cultura podemos ver que la mayoría de las mujeres se casan a muy temprana edad, muchas de ella son en su mayoría niñas, es decir, no alcanzan ni siquiera la mayoría de edad, la edad oscila entre los 12 y 18 años.

La informante 1 menciona que su cultura influye de manera negativa porque "Como mujer dicen que está mal que las mujeres estudiemos, nos prohíben estudiar porque no quieren que estudien las mujeres, dicen que solo van a buscar su marido en la escuela, así dicen algunas personas".

Otra de las situaciones en las que se ven limitadas las mujeres es en cuanto a la socialización con hombres, en la comunidad no es muy bien visto que una joven se encuentre platicando con un hombre. Así lo expresa la informante 4: "Mi cultura no es muy buena porque no quiere que tengamos amigos en la 
escuela y no quieren que salgamos a pasear con nuestros amigos"

La informante 10 por su parte nos menciona que "Mi cultura es negativa porque no nos dejan hacer muchas cosas porque somos niñas, nos prohíben muchas cosas me limitan que no debo hablarles a los niños y que no debo reír"

La informante 16 por su parte menciona "Mi cultura es negativa porque como mujeres nos prohíben a hacer ciertas cosas como: estudiar, participar entre otras, hasta se burlan y critican que estoy estudiado"

En su mayoría ellas perciben a su cultura con influencia negativa en su proceso educativo y en su vida en general, ya que ellas son limitadas en muchos de los aspectos de su vida. Aún bajo esa circunstancia ellas se sienten orgullosas de pertenecer a una etnia indígena.

El $19 \%$ de las informantes menciona que su cultura influye de manera positiva, porque se les ha permitido continuar estudiando, así lo expresa la informante 17 "Mi cultura si es positiva, porque como mujer estoy estudiando"

\section{ANÁLISIS}

La mayor parte de las informantes manifestó que su cultura no les permite desarrollarse como ellas quisieran ya que cultural y socialmente son muy limitadas en sus acciones, esto con relación a lo que es permitido para los hombres. Ellas no pueden hacer muchas de las cosas que hacen los hombres, como por ejemplo salir tarde para hacer un trabajo, irse acompañadas de un joven por las veredas o el camino hacia su casa, hablar u opinar frente a los hombres.

Si bien, la cultura es un elemento indispensable para darle continuidad a la sociedad, en ocasiones suele influir de manera negativa, citando a Vygotsky (1979), la cultura es el determinante primario del desarrollo individual. Los seres humanos somos los únicos que creamos cultura y es en ella donde nos desarrollamos y a través de la cultura los individuos adquieren el contenido de su pensamiento, dicho en otras palabras, la cultura es la que nos proporciona los medios para adquirir esos conocimientos. La cultura nos dice qué pensar y cómo pensar, nos da el conocimiento y la forma de construir ese conocimiento mientras estemos insertos en una sociedad.

Para las jóvenes estudiantes del centro educativo EMSaD 315, la cultura ha limitado su crecimiento y desarrollo personal, desde pequeñas están acostumbradas a no opinar, a no expresarse, a no aprender de una forma libre perpetuando con ello una cultura machista.

Cuando hablamos de empoderamiento, no estamos pensando en inclinar la balanza hacia el otro lado, pues no estaríamos propiciando la equidad. Cuando hablamos de empoderamiento se piensa en una redistribución del poder, de las oportunidades, de los espacios.

Respecto al empoderamiento de las mujeres y en este caso de las mujeres indígenas, constituye una estrategia para transformar estructuras e instituciones que refuerzan la discriminación de género y capacitar a las mujeres para que accedan a información y recursos clave para su desarrollo personal (Batliwala, 1997). Así también, para León (1997) el concepto de empoderamiento tiene una relación directa con el de autonomía, entendida como un proceso de negociación con otros espacios, entre ellos el Estado, y no como individualización y separación.

La Educación Media Superior es una vía para el empoderamiento de las jóvenes indígenas estudiantes. Es importante mencionar que el $100 \%$ de las jóvenes entrevistadas ven a la educación como una oportunidad viable para mejorar su calidad de vida, además de aprender y adquirir nuevos conocimientos la escuela 
también les genera la formulación de planes a futuro. A través de la escuela se les ha permitido conocer sus derechos y defenderse de prácticas abusivas por parte de algunos hombres miembros de su comunidad.

\section{CONCLUSIONES}

La educación debe garantizar en todo momento la equidad y eliminar las desigualdades educativas en las comunidades indígenas. Solís (2016), menciona que el verdadero objetivo de la educación es lograr la equidad de género; misma que no sólo significa incorporar a más niñas, adolescentes y mujeres adultas en los distintos niveles educativos, sino erradicar todas las formas de manifestación del sexismo en los procesos educativos.

El proceso educativo es de suma importancia para el desarrollo personal, académico y profesional de un individuo. Es difícil de creer que, en pleno siglo XXI continuemos encontrando prácticas discriminatorias hacia las mujeres. Por otra parte, el presente estudio confirma también el desarrollo de habilidades y competencias que generan sentido de superación en las jóvenes estudiantes.

Así también, se identificaron las percepciones de las jóvenes entrevistadas, tomando claridad del proceso de formación y haciendo explícito el sentimiento de exclusión generado por el machismo como parte de una cultura permanente en sus comunidades. Así también y de forma determinante, las jóvenes perciben a la escuela, media superior, como una oportunidad de empoderamiento, vindicación de género y superación personal.

\section{REFERENCIAS BIBLIOGRÁFICAS}

Araya U.S. (2001). La equidad de género en la educación. La Ventana Núm. 13, Disponible en: file:///C:/Users/PC1/ Downloads/Dialnet-LaEquidadDeGen eroEnLaEducacion-5202171.pdf

Batliwala, S. (1997). "El significado del empoderamiento de las mujeres: nuevos conceptos desde la acción", en León, $M$ (Ed.), Poder y empoderamiento de las mujeres, Bogotá, Tercer Mundo, pp.187214.

CONACPO, Consejo Nacional de Población (2010). Índice de marginación por localidad 2010.

Dietz, G. 2014 Educación intercultural en México. En: CPUe. Revista de Investigación Educativa, No. 18. Xalapa, Ver.: Universidad Veracruzana, cfr. cfr. http://revistas.uv.mx/index.php/ cpue/article/view/761

García, S.S. (2000). DE LA EDUCACIÓN INDÍGENA A LA EDUCACIÓN BILINGÜE INTERCULTURAL. La comunidad purhepecha, Michoacán, México Revista Mexicana de Investigación Educativa, vol. 9, núm. 20, enero-marzo, 2004, pp. 61-81 Consejo Mexicano de Investigación Educativa, A.C. Distrito Federal, México.

Instituto Nacional de Estadística y Geografía. Censo de población y vivienda (2010), Tabulados básicos. 
Lamas, Martha. (1996). Tomado de La Tarea, Revista de Educación y Cultura de la Sección 47 del SNTE. No. 8. Eneromarzo.

Lamas, Martha. (2003). El género la construcción cultural de la diferencia sexual. Edit Miguel Ángel Porrúa. México.

León, M (1997). "El empoderamiento en la teoría y práctica del feminismo", en León, M. (Ed.), Poder y empoderamiento de las mujeres, Bogotá, Tercer Mundo, pp.1-26.

Montero, MDC. (2000). Revista educación y ciencia. La mujer mexicana y su desarrollo educativo: Breve historia y perspectiva. Julio-Diciembre.

Sánchez, LM (2018). Empoderamiento educativo de las mujeres. Situación actual y líneas de política. Equidad de la Mujer. República de Colombia.

Scott JW. (2003). El género: una categoría útil para el análisis histórico en Martha Lamas (compiladora). El género la construcción cultural de la diferencia sexual. Edit Miguel Ángel Porrúa. México.

Solís SA. (2016). La perspectiva de género en la educación. En J.A. Trujillo Holguín y J.L. García Leos (coords.), Desarrollo profesional docente: reforma educativa, contenidos curriculares y procesos de evaluación (pp. 97-107), Chihuahua, México: Escuela Normal Superior Profr. José E. Medrano R. 